\title{
Synthesis and In Vitro Evaluation of Anticancer activity of Mannich Bases of Benzimidazole Derivatives
}

\author{
Pranika Kaur ${ }^{1}$, Sharad Wakode ${ }^{2}$ \\ Delhi Institute of Pharmaceutical Sciences and Research (DIPSAR), New Delhi, India
}

\begin{abstract}
Benzimidazole moiety is an important class of heterocycles used for synthesis of medicinal compounds. Mannich bases of Benzimidazole were synthesized. The structures were identified by determining their melting points, TLC, IR spectral analysis and ${ }^{1}$ HNMR spectrum. Anticancer activity was performed in vitro for screening of synthesised compounds by Sulfordamine B (SRB) assay. Cytotoxicity of compounds was evaluated for lung, prostrate, colon and breast tissue. Compound VIII a and VIII b showed 100\% cytotoxicity against colon cancer. The compound VI c showed $89 \%$ cytotoxicity against lung cancer. Against prostate cancer compound VIII a resulted in $73 \%$ cytotoxicity. The compound VIII a and VI a showed 94\%, 93\% cytotoxicity respectively against breast cancer.
\end{abstract}

Keywords: Mannich base, Heterocyclic, Benzimidazole, Anticancer activity, Cytotoxicity, Genotoxicity

\section{Introduction}

In recent years, cancer has become a major killer and challenge to the world chemists. It is a disease in which cells divide and grow uncontrollably, consuming energy and losing both structure and function due to an inability to adequately differentiate [1]. The most prevalent cancers are lung, breast, prostrate, colon cancer. In spite of wide anticancer drugs available, it remains as a deadly disease. Anticancer drugs act either by killing the cancer cells or modifying their growth. But, such chemotherapy is often associated with cytotoxicity, genotoxicity to normal cells together with development of resistance [2]. Therefore; medicinal chemists are indulged in continuous research for screening of novel and safe anti-cancer agents.

Benzimidazole is a well-established potent anti-cancer molecule. The Imet 3393 is commercially available benzimidazole based anticancer drug [3]. Benzimidazole is a heterocyclic compound and important intermediate in organic reactions. In cancer therapy, resistance to wide range of unrelated drugs may occur after resistance to a single agent has developed. Multiple drug resistance is lack of expected therapeutic response to several disease-specific pharmaceutical agents [4]. Highly drug resistant tumour cells limit the success rate of cancer chemotherapy [5]. To combat this, novel compounds are needed to solve the problem of MDR therefore, compounds are formed by its modification through different chemical reactions to enhance its biological activity. In Pharmaceutical chemistry, Benzimidazole moieties are being developed as DNA minor grove binding agents that have significant anti-tumor activity [6]. Mannich Reaction is very useful for such modification of Benzimidazole molecule and it forms Mannich bases. Mannich reaction is the C-C bond forming condensation reaction of ammonia, primary or secondary amine, formaldehyde and compound containing at least one hydrogen atom of pronounced reactivity [7]. Mannich bases are versatile and reactive intermediates and, therefore, easily converted into other molecules In addition, Mannich bases of Benzimidazole are known to display anti-cancer activity. Mannich bases, derivatives of the various heterocycles, show antiproliferative activity in vitro against human tumor cell lines [8]. Based on these facts, it is worthwhile to synthesize mannich bases with enhanced anti-cancer activity.

\section{Materials and Method}

The chemicals to synthesise compound were procured from Merck, Lobachemie and were synthesised by using parallel synthesiser. The melting points of the synthesised compounds were determined by visual melting point apparatus. Reaction and purity of the compounds were analysed by use of thin layer chromatography (Chloroform: Methanol; 9:1). The IR spectra was recorded on ATR spectrophotometer. 1HNMR spectra was recorded at $300 \mathrm{MHz}$ using DMSO as solvent and TMS as internal standard.

\begin{tabular}{|c|c|}
\hline Compounds & \\
\hline $\mathrm{VI} \mathrm{a}$ & \\
\hline $\mathrm{VI} \mathrm{b}$ & \\
\hline $\mathrm{VI} \mathrm{c}$ & \\
\hline $\mathrm{VI} \mathrm{d}$ & \\
\hline $\mathrm{VIII} \mathrm{a}$ & \\
\hline $\mathrm{VIII} \mathrm{b}$ & \\
\hline
\end{tabular}




\section{International Journal of Science and Research (IJSR)}

ISSN (Online): 2319-7064

Index Copernicus Value (2013): 6. 14 | Impact Factor (2015): 6. 391

\subsection{Synthetic Procedures}<smiles>[R]c1nc2ccccc2[nH]1</smiles>

Substituted carboxylic acid : formic acid,benzoic acid,2-chlorobenzoic acid, 4-chlorobenzoic acid,3-nitrobenzoic acid

STEP 2

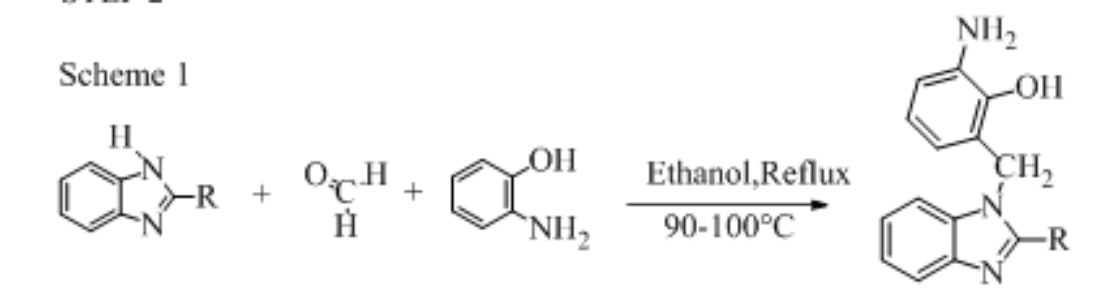

Benzimidazole Formaldehyde o-Aminophenol

Mannich base

(III a-e)

(IV)

(V)

(VI a-d)

STEP 2
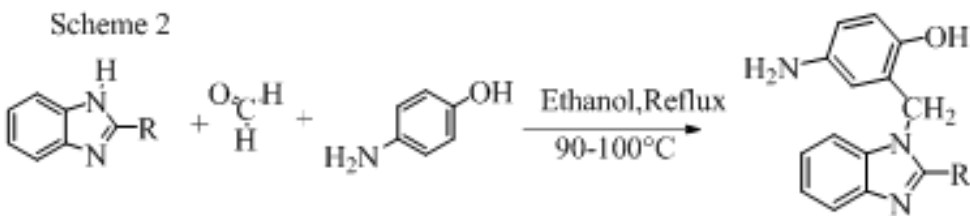

Benzimidazole

Formaldehyde p-Aminophenol

Mannich base

(III a-e)

(IV)

(VII)

(VIII a-c)

\section{For synthesis of Benzimidazole[9]}

In parallel synthesizer, ortho phenylenediamine $(0.02$ mole $)$ was refluxed with formic acid, benzoic acid and its derivatives $(0.02$ mole $)$ in the presence of $4 \mathrm{~N}$ hydrochloric acid $(4 \mathrm{~N} \mathrm{HCl})$ for $8-9$ hrs at temperature $140{ }^{\circ} \mathrm{C}$, rpm 800.The completion of the reaction was checked by TLC (chloroform: methanol 9:1). On completion, $10 \% \mathrm{NaOH}$ $(\mathrm{w} / \mathrm{v})$ was gradually added until the reaction mixture turns alkaline. To obtain precipitate, reaction mixture was cooled in ice bath and allowed to stand for $5 \mathrm{~min}$. The product was filtered, dried and recrystallized from ethanol.

\section{For synthesis of Mannich bases}

Using ortho and para aminophenol as substrate (Scheme 1 and Scheme 2) 0.005 mole of o-aminophenol or paminophenol and 0.005 mole of formaldehyde (formalin) were added to the solution of substituted Benzimidazole $(0.005$ mole $)$ in $10 \mathrm{ml}$ of ethanol in $250 \mathrm{ml}$ round bottom flask. The mixture was then refluxed for 8-10 hour on parallel synthesizer at $90-100{ }^{\circ} \mathrm{C}$. On cooling, reaction mixture was poured on crushed ice. The lump mass of precipitate was obtained which was then washed with ether, filtered and dried. The solid precipitate was isolated and dried in air.

\subsection{Biological Activity}

Anticanceractivity[10]

The in vitroanticancer activity was carried out at CSIR Jammu. All synthesised compounds were tested for In vitro cytotoxicity against four different cancer cell lines including Lung (A549), Prostate (PC-3), Colon (HCT-116) and Breast (MCF-7) cell lines. The compounds were tested at $100 \mu \mathrm{M}$ concentration.

Sulforhodamine B (SRB) assay was performed to determine In vitro cytotoxicity. Four human cell lines of various tissue origin were used to evaluate the cytotoxic activity of compounds. In this method, cell suspension was seeded into 96 well flat bottom plates and incubated for $24 \mathrm{~h}$. Test compounds at $100 \mu \mathrm{M}$ were added after $24 \mathrm{~h}$ incubation. Further, after $48 \mathrm{~h}$ incubation, cells were fixed with ice cold TCA for $1 \mathrm{~h}$ at $4{ }^{\circ} \mathrm{C}$. After $1 \mathrm{~h}$, the plates were washed five times with distilled water and allowed to air dry followed by addition of $100 \mu \mathrm{l}$ of $0.4 \%$ SRB dye for $0.5 \mathrm{~h}$ at room temperature. Plates were then washed with $1 \% \mathrm{v} / \mathrm{v}$ acetic acid to remove the unbound SRB dye. The bound dye was solubilised by adding $100 \mu \mathrm{l}$ of $10 \mathrm{mM}$ Tris buffer to each

\section{Volume 5 Issue 5, May 2016}




\section{International Journal of Science and Research (IJSR) \\ ISSN (Online): 2319-7064}

Index Copernicus Value (2013): 6. 14 | Impact Factor (2015): 6. 391

well. Then the plates were put on shaker for $5 \mathrm{~min}$ to solubilize the dye completely. Finally the reading was taken at $540 \mathrm{~nm}$ on microplate reader. The cytotoxic screening data of compounds at $100 \mu \mathrm{M}$ concentration is provided in table no. 2

\section{Results and Discussion}

\subsection{Chemistry}

The benzimidazole derivatives in above scheme were synthesised by the methods reported in the earlier literature [11]. The physical characterization of Mannich base of Benzimidazole derivatives are presented in table no. 1

Table 1: Physical characterization of Mannich base of Benzimidazole derivatives

\begin{tabular}{|c|c|c|c|c|c|}
\hline $\begin{array}{c}\text { S. } \\
\text { No. }\end{array}$ & Compound & $\begin{array}{c}\text { Molecular } \\
\text { formula }\end{array}$ & Rf & \%yield & $\begin{array}{c}\text { Melting } \\
\text { Point }\left({ }^{\circ} \mathrm{C}\right)\end{array}$ \\
\hline 1 & VI a & $\mathrm{C}_{14} \mathrm{H}_{13} \mathrm{~N}_{3} \mathrm{O}$ & 0.774 & $54 \%$ & $185-188$ \\
\hline 2 & VI b & $\mathrm{C}_{21} \mathrm{H}_{17} \mathrm{ClN}_{2} \mathrm{O}$ & 0.6 & $68 \%$ & $235-239$ \\
\hline 3 & VI c & $\mathrm{C}_{20} \mathrm{H}_{16} \mathrm{ClN}_{3} \mathrm{O}$ & 0.9 & $75 \%$ & $275.5-278$ \\
\hline 4 & VI d & $\mathrm{C}_{20} \mathrm{H}_{16} \mathrm{~N}_{4} \mathrm{O}_{3}$ & 0.791 & $55 \%$ & $213-215$ \\
\hline 5 & VIII a & $\mathrm{C}_{20} \mathrm{H}_{16} \mathrm{ClN}_{3} \mathrm{O}$ & 0.75 & $72 \%$ & $240-245$ \\
\hline 6 & VIII b & $\mathrm{C}_{20} \mathrm{H}_{16} \mathrm{ClN}_{3} \mathrm{O}$ & 0.821 & $69 \%$ & $274.9-277$ \\
\hline 7 & VIII c & $\mathrm{C}_{20} \mathrm{H}_{16} \mathrm{~N}_{4} \mathrm{O}_{3}$ & 0.76 & $42 \%$ & $210-211$ \\
\hline
\end{tabular}

Spectral Analysis OF Synthesised Compounds

1. 2-amino-6-(1H-benzimidazole-1-ylmethyl)phenol (VI a) ATR : $\mathrm{OH}\left(3539.06 \mathrm{~cm}^{-1}\right), \mathrm{NH}_{2}\left(3401.01 \mathrm{~cm}^{-1}\right)$, $\mathrm{CH}_{2}\left(2920.50,2853.04 \mathrm{~cm}^{-1}\right), \mathrm{Ar} \mathrm{C}=\mathrm{C}\left(1600.11 \mathrm{~cm}^{-1}\right)$, $\mathrm{CH}_{2}$ bend(1475. $\left.24 \mathrm{~cm}^{-1}\right)$, ortho substitution $(747.75 \mathrm{~cm}$ $\left.{ }^{1}\right),{ }^{1} \mathrm{HNMR}(300 \mathrm{MHz}, \mathrm{DMSO}): \delta 8.43(\mathrm{~s}, 1 \mathrm{H}, \mathrm{Ar}-\mathrm{H}), 7$. 70(ddd, 2H, Ar-H), 7. 32(td, 1H, Ar-H), 7. 20(td, 1H, ArH), 6. 74(ddt, 1H, Ar-H), 6. 68(t, 1H, ArH), 6. 43(dd, 1H), 5. 93(s, 1H, OH), 5. 34(d, $\left.2 \mathrm{H}, \mathrm{CH}_{2}\right), 3.18(\mathrm{~s}, 2 \mathrm{H}$, $\mathrm{NH}_{2}$ )

2. 2-amino-6-\{[2-(4-chlorophenyl)-1H-benzimidazole-1-yl] methyl $\}$ phenol (VI b)

ATR : $\mathrm{OH}\left(3531.09 \quad \mathrm{~cm}^{-1}\right), \quad \mathrm{NH}_{2}\left(3413.21 \quad \mathrm{~cm}^{-1}\right)$, $\mathrm{CH}_{2}$ (2889. $\left.38 \mathrm{~cm}^{-1}\right)$, Ar $\mathrm{C}=\mathrm{C} \quad\left(1603.44, \mathrm{~cm}^{-1}\right), \mathrm{CH}_{2}$ bend(1444. $\left.36 \mathrm{~cm}^{-1}\right)$, ortho substitution $\left(750.34 \mathrm{~cm}^{-1}\right)$, R$\mathrm{Cl}\left(648.32 \mathrm{~cm}^{-1}\right),{ }^{1} \mathrm{H}$ NMR(300 MHz, DMSO $): \delta 7.84-$ 7. $76(\mathrm{~m}, 1 \mathrm{H}, \mathrm{Ar}-\mathrm{H})$, 7. 59-7. 53(m, 1H, Ar-H), 7. 52-7. 42(m, 2H, Ar-H), 7. 35 7. 28(m, 1H, ArH), 6. 86(ddt, 1H, ArH $)$ 6. 72(t, 1H, ArH), 6. 51(dd, 1H, ArH), 5. 93(s, 1H, $\mathrm{OH}), 5.34\left(\mathrm{~d}, 1 \mathrm{H}, \mathrm{CH}_{2}\right)$

3. 2-amino-6- $\{[2-(2$-chlorophenyl $) 1 \mathrm{H}$-benzimidazole-1yl]methyl phenol(VI c)

ATR : $\mathrm{OH}\left(3505.22 \mathrm{~cm}^{-1}\right), \mathrm{NH}_{2}\left(3443.09 \mathrm{~cm}^{-1}\right)$, $\mathrm{CH}_{2}$ (2978. 21, 2884. 09 $\left.\mathrm{cm}^{-1}\right), \mathrm{Ar} \mathrm{C}=\mathrm{C}(1610.06, \mathrm{~cm}-1)$, $\mathrm{CH}_{2}$ bend(1441. $\left.28 \mathrm{~cm}^{-1}\right)$, ortho substitution $\left(655.47 \mathrm{~cm}^{-}\right.$ $\left.{ }^{1}\right),{ }^{1} \mathrm{HNMR}(300 \mathrm{MHz}, \mathrm{DMSO}): \delta 7.84-7.78(\mathrm{~m}, 1 \mathrm{H}$, Ar-H), 7.58 (td, 2H, Ar-H), 7. $52-7.44$ (m, 1H, Ar-H), 7. $41-7.32$ (m, 2H, Ar-H), 7. 35 - 7.27 (m, 2H, Ar-H), 6. 85 (ddt, 1H, Ar-H), 6.70 (t, 1H, Ar-H), 6. 44 (dd, 1H, Ar-H), 5. 93 (s, 1H, OH), 5. 34 (d, 2H, $\left.\mathrm{CH}_{2}\right), 3.17$ (s, $\left.2 \mathrm{H}, \mathrm{NH}_{2}\right)$.

4. 2-amino-6-\{[2-(3-nitrophenyl)-1H-benzimidazole-1-yl] methyl \}phenol (VI d)

ATR : $\mathrm{OH}\left(3544.74 \quad \mathrm{~cm}^{-1}\right), \quad \mathrm{NH}_{2}\left(3380.33 \quad \mathrm{~cm}^{-1}\right)$, $\mathrm{CH}_{2}\left(2991.06,2718.76 \mathrm{~cm}^{-1}\right), \mathrm{Ar} \mathrm{C}=\mathrm{C}(1603.00 \mathrm{~cm}-1)$,
$\mathrm{CH}_{2}$ bend $\left(1475.96 \mathrm{~cm}^{-1}\right)$, ortho substitution $(753.89 \mathrm{~cm}$ $\left.{ }^{1}\right), \quad \mathrm{R}-\mathrm{NO}_{2}\left(1362.42 \quad \mathrm{~cm}^{-1}\right),{ }^{1} \mathrm{HNMR}(300 \mathrm{MHz}$, DMSO): $\delta 8.52(\mathrm{t}, 1 \mathrm{H}, \mathrm{Ar}-\mathrm{H}), 8.28(\mathrm{ddt}, 2 \mathrm{H}, \mathrm{Ar}-\mathrm{H}), 7.84-$ 7. 76(m, 1H, ArH $), 7.62(\mathrm{t}, 1 \mathrm{H}, \mathrm{Ar}-\mathrm{H}), 7.52-7.44(\mathrm{~m}$, 1H, Ar-H), 7. 36-7. 27(m, 2H, Ar-H), 6. 63-6. 54(m, 2H, Ar-H), 6. 28(dd, 1H, Ar-H), 5. 93(s, 1H, OH), 5. 34(d, $\left.2 \mathrm{H}, \mathrm{CH}_{2}\right), 3.89\left(\mathrm{~s}, 2 \mathrm{H}, \mathrm{NH}_{2}\right)$

5. 4-amino-2-\{[2-(4-chlorophenyl)-1H-benzimidazole-1yl]methyl $\}$ phenol(VIII a)

ATR: $\mathrm{OH}\left(3534.13 \quad \mathrm{~cm}^{-1}\right), \quad \mathrm{NH}_{2}\left(3415.29 \mathrm{~cm}^{-1}\right)$, $\mathrm{CH}_{2}$ (2978. 04, 2887. 34 $\left.\mathrm{cm}^{-1}\right), \mathrm{Ar} \mathrm{C}=\mathrm{C}(1610.16 \mathrm{~cm}-1)$, $\mathrm{CH}_{2}$ bend $\left(1452.80 \mathrm{~cm}^{-1}\right)$, para substitution $\left(831.36 \mathrm{~cm}^{-1}\right)$, ${ }^{1} \mathrm{HNMR}(300 \mathrm{MHz}, \mathrm{DMSO}): \delta 7.84-7.76(\mathrm{~m}, 1 \mathrm{H}, \mathrm{Ar}-$ H), 7. $58-7.53(\mathrm{~m}, 1 \mathrm{H}, \mathrm{Ar}-\mathrm{H}), 7.52-7.42(\mathrm{~m}, 2 \mathrm{H}, \mathrm{Ar}-$ H), 7. $35-7.28(\mathrm{~m}, 1 \mathrm{H}, \mathrm{Ar}-\mathrm{H}), 6.68(\mathrm{dt}, 1 \mathrm{H}, \mathrm{Ar}-\mathrm{H}), 6$. $47-6.36(\mathrm{~m}, 1 \mathrm{H}, \mathrm{Ar}-\mathrm{H}), 6.10(\mathrm{~s}, 1 \mathrm{H}, \mathrm{OH}), 5.34(\mathrm{~d}, 2 \mathrm{H}$, $\left.\mathrm{CH}_{2}\right), 3.94$ (s, 2H, $\mathrm{NH}_{2}$ ).

6. 4-amino-2-\{[2-(2-chlorophenyl)-1H-benzimidazole-1yl]methyl $\}$ phenol(VIII b)

ATR : $\mathrm{OH}\left(3555.01 \quad \mathrm{~cm}^{-1}\right), \quad \mathrm{NH}_{2}$ (3441. $\left.05 \quad \mathrm{~cm}^{-1}\right)$, $\mathrm{CH}_{2}\left(2977.81,2886.41 \mathrm{~cm}^{-1}\right), \mathrm{Ar} \mathrm{C}=\mathrm{C}(1681.41 \mathrm{~cm}-1)$, $\mathrm{CH}_{2}$ bend $\left(1460.91 \mathrm{~cm}^{-1}\right)$, para substitution $\left(833.37 \mathrm{~cm}^{-1}\right)$, ${ }^{1} \mathrm{HNMR}(300 \mathrm{MHz}, \mathrm{DMSO}): \delta 7.84-7.76(\mathrm{~m}, 1 \mathrm{H}, \mathrm{Ar}-$ H), 7. 58 (ddd, 2H, Ar-H), 7. 52 - 7.44 (m, 1H, Ar-H), 7. $41-7.32(\mathrm{~m}, 2 \mathrm{H}, \mathrm{Ar}-\mathrm{H}), 7.35-7.27$ (m, 2H, Ar-H), 6. $72(\mathrm{dt}, 1 \mathrm{H}, \mathrm{Ar}-\mathrm{H}), 6.46-6.35(\mathrm{~m}, 2 \mathrm{H}, \mathrm{Ar}-\mathrm{H}), 6.08(\mathrm{~s}$, $1 \mathrm{H}, \mathrm{OH}), 5.34\left(\mathrm{~d}, 2 \mathrm{H}, \mathrm{CH}_{2}\right), 3.94\left(\mathrm{~s}, 2 \mathrm{H}, \mathrm{NH}_{2}\right)$

7. 4-amino-2-\{[2-(3-nitrophenyl)-1H-benzimidazole-1yl]methyl $\}$ phenol (VIII c)

ATR : $\mathrm{OH}\left(3537.64 \mathrm{~cm}^{-1}\right), \quad \mathrm{NH}_{2}\left(3413.39 \mathrm{~cm}^{-1}\right)$, $\mathrm{CH}_{2}\left(2919.97,2855.19 \mathrm{~cm}^{-1}\right), \mathrm{Ar} \mathrm{C}=\mathrm{C}(1605.05 \mathrm{~cm}-1)$, $\mathrm{CH}_{2}$ bend(1478. $\left.35 \mathrm{~cm}^{-1}\right), \mathrm{R}-\mathrm{NO}_{2}\left(1377.56 \mathrm{~cm}^{-1}\right)$ para substitution (828. $\left.75 \mathrm{~cm}^{-1}\right),{ }^{1} \mathrm{HNMR}(300 \mathrm{MHz}, \mathrm{DMSO})$ :

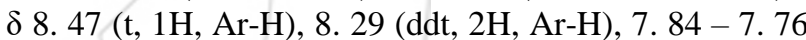
$(\mathrm{m}, 1 \mathrm{H}, \mathrm{Ar}-\mathrm{H}), 7.63(\mathrm{t}, 1 \mathrm{H}, \mathrm{Ar}-\mathrm{H}), 7.52-7.44(\mathrm{~m}, 1 \mathrm{H}$, Ar-H), 7. $36-7.27$ (m, 2H, Ar-H), 6. $54-6.48$ (m, 2H, Ar-H), 6. 36 (s, 1H, Ar-H), 6. 30 (dd, 1H, OH), 5. 34 (d, $\left.2 \mathrm{H}, \mathrm{CH}_{2}\right), 3.93\left(\mathrm{~s}, 2 \mathrm{H}, \mathrm{NH}_{2}\right)$

\subsection{Anticancer Activity}

Compound VIII a and VIII b showed $100 \%$ cytotoxicity against colon cancer. The compound VI c showed $89 \%$ cytotoxicity against lung cancer. Against prostate cancer compound VIII a resulted in $73 \%$ cytotoxicity. The compound VIII a and VI a showed 94\%, 93\% cytotoxicity respectively against breast cancer.

Table 2: In vitro Cytotoxicity against human cancer cell lines

\begin{tabular}{|c|c|c|c|c|c|}
\hline \multicolumn{2}{|c|}{ Tissue } & Lung & Prostate & Colon & Breast \\
\hline \multicolumn{2}{|c|}{ CELL lines } & A549 & PC-3 & HCT-116 & MCF-7 \\
\hline CODE & CONC(100 $\mu \mathrm{M})$ & & & & \\
\hline VI a & 100 & 75 & 32 & 87 & 93 \\
\hline VI b & 100 & 70 & 65 & 90 & 81 \\
\hline VI c & 100 & 89 & 37 & 71 & 75 \\
\hline VI d & 100 & 22 & 23 & 10 & 47 \\
\hline VIII a & 100 & 83 & 73 & 100 & 94 \\
\hline VIII b & 100 & 77 & 39 & 100 & 89 \\
\hline VIII c & 100 & 28 & 30 & 8 & 50 \\
\hline
\end{tabular}




\section{International Journal of Science and Research (IJSR) \\ ISSN (Online): 2319-7064}

Index Copernicus Value (2013): 6. 14 | Impact Factor (2015): 6. 391

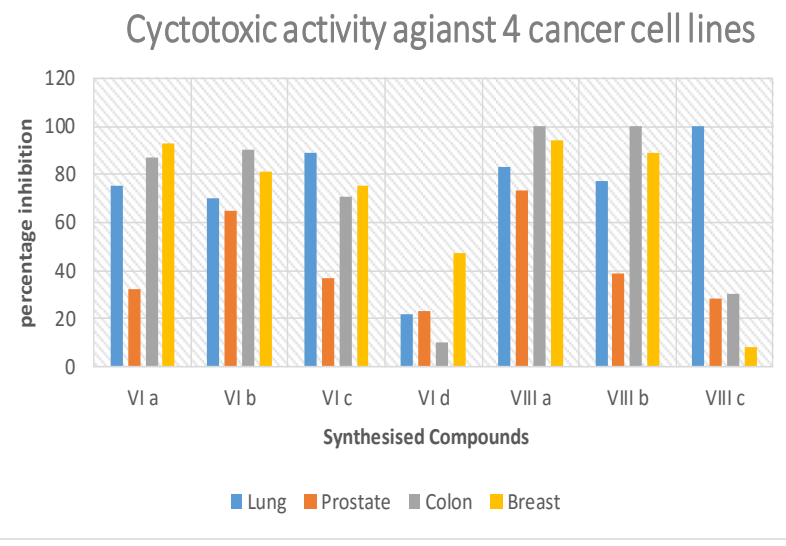

\section{Conclusion}

A series of Mannich base derivatives were synthesised and screened for biological activity. So, it can be concluded that the designed compounds are potent anticancer agent.

The diverse aspects clearly show the high potential of Mannich bases and some compounds showed significant anticancer $(100 \%)$ activity. In summary, all the Mannich bases of Benzimidazole derivatives showed promising anticancer activity. This work will hopefully be used for further development of potential drugs.

\section{Acknowledgement}

The author is thankful to Dr. Sharad Wakode, associate professor, Delhi Institute of Pharmaceutical Sciences and Research (DIPSAR) for accomplishment of the research work and also grateful to CSIR Jammu for providing anticancer data.

\section{References}

[1] Foye, W., Lemke, T. and Williams, D. (2008). Foye's principles of medicinal chemistry. Philadelphia: Lippincott Williams \& Wilkins, p. 1199.

[2] Aydemir, N. and Bilaloğlu, R. (2003). Genotoxicity of two anticancer drugs, gemcitabine and topotecan, in mouse bone marrow in vivo. Mutation Research/Genetic Toxicology and Environmental Mutagenesis, 537(1), pp. 43-51

[3] Khokra, S. and Choudhary, D. (2011). Benzimidazole An Important Scaffold In Drug Discovery. Asian Journal of Biochemical and Pharmaceutical Research, 1(3), pp. 476-486.

[4] Venes, D. (2006). Taber's Cyclopedic Medical Dictionary. 20th ed. New Delhi, Jaypee Brothers, p. 1397.

[5] GanesanSubramaniapillai, S. (2013). Mannich Reaction:A versatile and convenient approach to bioactive skeletons. J. Chem. Sci., 125(3), p. 467.

[6] SR reddy, B. (2013). A green synthesis of benzimidazoles. Indian Journal of chemistry, 52B, pp. 1152-1156

[7] Blicke, F. (1942.). The Mannich reaction. chapter 10, pp. 303-341.
[8] Nowicka, A. (2015). Synthesis and In vitro antiproliferative activity of novel 2Arylideneaminobenzimidazole derivatives. Acta Poloniae Pharmaceutica - Drug Research, 72(5), pp. 951-963.

[9] Divya, B. (2012). Synthesis and characterization of novel Benzimidazole derivatives IJPBS, 2, pp. 143-149.

[10] Singh, B., Guru, S., Kour, S., Jain, S., Sharma, R., Sharma, P., Singh, S., Bhushan, S., Bharate, S. and Vishwakarma, R. (2013). Synthesis, antiproliferative and apoptosis-inducing activity of thiazolo[5, 4d]pyrimidines. European Journal of Medicinal Chemistry, 70, pp. 864-874

[11] Divya, B. (2012). Synthesis and characterization of novel Benzimidazole derivatives IJPBS, 2, pp. 143-149

$=$

